\title{
The Relationship between Trunk Leg Ratio and Peak Expiratory Flow Rate in 200 Level MBBS/BDS Students of Bayero University Kano
}

\author{
Jibril I. Adama ${ }^{1^{*}}$, Salisu A. Ibrahim ${ }^{1}$, Y. Abdulmumin ${ }^{2}$ and Mukhtar F. Zahra ${ }^{1}$ \\ ${ }^{1}$ Department of Human Physiology, Bayero University, Kano, Nigeria. \\ ${ }^{2}$ Department of Biochemistry, Kano University of Science and Technology, Wudil, Kano, Nigeria.
}

Authors' contributions

This work was carried out in collaboration between all authors. Author JIA designed the study, performed the statistical analysis, wrote the protocol and wrote the first draft of the manuscript.

Authors YA and SAI managed the analyses of the study. Author MFZ managed the literature searches. All authors read and approved the final manuscript.

Article Information

DOI: $10.9734 / A R R B / 2018 / v 30 i 630032$

Editor(s):

(1) Dr. Moacir Marocolo, Physiology and Human Performance Research Group, Department of Physiology, Federal University of Juiz de Fora, Brazil.

(2) Dr. George Perry, Dean and Professor of Biology, University of Texas at San Antonio, USA.

Reviewers:

(1) Maria Justine, Universiti Teknologi MARA, Malaysia.

(2) Nuhu Sambo, Baze University Abuja, Nigeria

(3) Itamar Souza de Oliveira Junior, Federal University of São Paulo, Brazil. Complete Peer review History: http://www.sdiarticle3.com/review-history/47410

Original Research Article

Received 23 November 2018

Accepted 10 March 2019

Published 18 March 2019

\begin{abstract}
Background: Being an important physiological and clinical tool in assessing respiratory conditions, it is common knowledge that Peak expiratory flow rate (PEFR) may be affected by some factors affecting the normal function of the respiratory system. Such factors include the body constitution such as height, built, sex, age etc.; The trunk-leg ratio (TLR) was used in apparently normal young adults. A better understanding of the association between Trunk-leg ratio and PEFR may identify those with elevated risk of respiratory diseases.

Methods: The study was an analytical cross-sectional design, involving 83 Level $200 \mathrm{MBBS} / \mathrm{BDS}$ students of Bayero University, Kano. There were a total of 39 males and 44 females. A peak flow meter and a measuring tape to scale were used to measure the PEFR, trunk length and leg length respectively. A questionnaire was used to determine any history of cardio-respiratory disease $A$ consent of the participants and ethical approval was obtained before the commencement of the study.
\end{abstract}


Results: The males had higher PEFR value $(491.79 \pm 67.19 \mathrm{~L} / \mathrm{min})$ while the females had a lower PEFR of $366.82 \pm 43.28 \mathrm{~L} / \mathrm{min}$ and the difference was statistically significant. The males had higher values of trunk length, leg length and TLR and there was no significant different between that male and female trunk length ratio. There was A significant correlation between PEFR and leg length indicating that the longer the leg length, the higher the PEFR.

Conclusion and Recommendation: Significant correlation was found between the TLR, which is an anthropometric parameter and the PEFR which is an important diagnostic tool in determination of some types of respiratory diseases. This relationship signifies that the taller the person, irrespective of the trunk length, the higher the PEFR. It is recommended that further studies should be explored involving other anthropometric parameters like trunk-leg volume in future studies.

Keywords: PEFR; trunk length; leg length; trunk-leg ratio; respiratory diseases.

\section{INTRODUCTION}

The Peak expiratory flow rate (PEFR; also known as a peak flow) is the maximal rate that a person can exhale during a short maximal expiratory effort after a full inspiration, indicating full lung inflation. It actually depends on voluntary respiratory effort of the individual and the strength of the respiratory muscles [1]. Maximal airflow occurs during the effort-dependent portion of the expiratory maneuver, so low values may be caused by a less than maximal effort rather than by airway obstruction [2]. Nevertheless, the ease of measuring peak flow rate with an inexpensive small portable device has made it popular as a means of following the degree of airway obstruction in patients with asthma and other pulmonary conditions [1]. Forced expiratory volume over 1 second $\left(\mathrm{FEV}_{1}\right)$ is a dynamic measure of flow used in formal spirometry. It represents a truer indication of airway obstruction than PEFR. Although the latter usually correlates well with $\mathrm{FEV}_{1}$, this correlation decreases in patients with asthma as airflow diminishes [3]. PEFR monitoring can be accurately performed by most patients older than 5 years. It is most commonly measured by a portable flow gauge device but may also be obtained by a transducer that converts flow to electric output during spirometry (pneumotachometer) [4]. The most frequent use of PEFR measurement is in home monitoring of asthma, where it can be beneficial in patients for both short- and long-term monitoring. When properly performed and interpreted, peak flow rate measurement can provide the patient and the clinician with objective data upon which to base therapeutic decisions [5]. There are conflicting data regarding the efficacy of peak flow rate monitoring for improving asthma outcome [6].

Trunk-leg ratio could be a potential indicator for identifying the elevated risk of cardio-respiratory diseases. From a public health perspective, a better understanding of the association between Trunk-leg ratio and other parameters may provide an opportunity for the identification those with elevated risk of cardio-respiratory diseases [7].

Body shape is one of the known risk factors for morbidity in cardio-respiratory diseases, which are major global health problems associated with reduced life span, increased risk of mortality and significant financial burdens on the individuals and health care systems. Some well-known measures of body shape such as body mass index, (BMI) and waist circumference, (WC) are crude indicators of adipose tissue distribution but are globally recognized to be associated with risk of cardio-respiratory diseases [8].

Trunk-Leg ratio (TLR) or Leg-to-trunk ratio (LTR) is the ratio of leg length and trunk length, and a larger value of TLR indicates a higher leg length for a given body height. Leg length is calculated as the difference between height and sitting height, and TLR is obtained by dividing leg length by sitting height. Leg length is a marker of environmental influences on childhood growth before puberty as, up until puberty, height increases are in greater part attributable to leg growth [9]. Furthermore, secular increases in height, representing improvements in the nutritional status of populations, appear to arise more from increases in leg length, rather than trunk growth [10]. Therefore, PEFR serves as a measure for both restrictive and obstructive lung diseases with Trunk-leg ratio serving as an anthropometric measure that may help in their determination. Also, many researches have been conducted to determine the relationship between TLR and cardiovascular risks, very few explored PEFR especially in this part of the world [11]. This study was aimed to evaluate the relationship between trunk leg ratio and peak expiratory flow 
rate in 200 level MBBS/BDS students of Bayero University Kano.

\section{METHODS}

\subsection{Sampling and Data Collection}

A total of Eighty-three (83) MBBS/BDS 200 level students as the sample size was estimated from statistical table for sample size determination A non-probability sampling technique was used in such a way that only the students who give their consent and were willing to participate in the study were used. The procedure was explained to the volunteering subjects and consent form was given to them so as to sign. A measuring tape was used in measuring the Trunk length and Leg length and a peak flow meter was used in measuring Peak Expiratory Flow Rate.

\subsection{Determination of Trunk-Leg Ratio}

In this study, the trunk-leg ratio was determined as follows; the subject was asked to stand in an anatomical position and a measuring tape was used to measure the trunk-length which was measured from the shoulder to the summit of the iliac crest. With the subject still in anatomical position, the leg length was measured which was gotten from the summit of the iliac crest down to the floor. The Trunk-leg ratio was gotten by dividing the trunk length with the leg length [12].

\subsection{Determination of Peak Expiratory Flow Rate}

PEFR (L/min) was evaluated with the brand name device Mini Wright (Clement Clarke International Ltd., Essex, England) with a range of 60 to $900 \mathrm{~L} / \mathrm{min}$ [13]. The PEFR was obtained by a forced exhalation maneuver beginning with a maximum inhalation (equal to a spirometric test). Following the directions of Quanjer et al. [14], subjects were evaluated in a standing position without bending the neck. Prior to the evaluation, the device was described to the adolescent subjects, and they were allowed to practice the exercise twice (familiarization). Afterwards, the assessment was carried out with the highest value recorded of the three attempts. The Technical Error of Measurement (TEM) was less than $2 \%$ for both sexes. The PEFR was classified as lowest (first tertile), middle (second tertile), and highest (third tertile). The participants were asked to stand erect holding the medium
Wright Peak Expiratory Flow Meter with one hand and was asked to maximally inspire atmospheric air and then wrap the mouth around the mouth piece of the Peak Flow Meter then use the other hand to close the nose then expire maximally into the Peak Flow Meter until the air filling the lungs was expired completely [15].

\subsection{Statistical Analysis}

The data collected was expressed as mean \pm standard deviation and the statistical analysis was done using Statistical Package for Social Sciences (SPSS) version 20.0 and excels 2013 for windows. Both inferential and descriptive statistics was used to examine the outcome of the study. Pearson's Moment of Correlation and independent sample t-test were used in determining the relationship between LTR and PEFR. A p-value of $<0.05$ will be considered statistically significant in all the calculations.

\section{RESULTS AND DISCUSSION}

A total of 83 students; both males (39) and females (44) from first year of MBBS/BDS of Bayero University Kano were recruited for the study. All of whom were in the adolescent age group 18 to 25 years.

A relationship between TLR and PEFR was sought for and this research is not in contrast with the findings of previous studies [15] who both found a relationship between Trunk-leg ratio and PEFR. A significant positive correlation between leg length and PEFR was found in this research which means that the longer the legs of an individual, the higher the PEFR value (Tables 1 and 2).

Majority of the parameters of the males were higher than those of the females, significantly. Except for the trunk length in which the difference is not significant. This difference is purely constitutional, which is due to the difference in anthropometric parameters between males and females. The difference in PEFR could possibly be due to stronger muscles in males.

The result of this research study found that there was no any significant relationship between Trunk-leg ratio and PEFR (Table 3). It may be a useful predictor when dealing with mixed ethnic origins due to the fact that blacks have lower Trunk-to-leg ratio [16]. 
Table 1. Descriptive statistics of anthropometric variables of the mean value of PEFR, trunk length, Leg length and trunk-leg ratio among the participants

\begin{tabular}{lll}
\hline Variables & Number $($ No) & Mean \pm STD \\
\hline PEFR $(\mathrm{L} / \mathrm{min})$ & 83 & $425.54 \pm 83.74$ \\
Trunk length $(\mathrm{cm})$ & 83 & $16.93 \pm 1.606$ \\
Leg length $(\mathrm{cm})$ & 83 & $39.51 \pm 2.62$ \\
Trunk-leg ratio & 83 & $0.43 \pm 0.51$ \\
\hline
\end{tabular}

Table 2. Comparison of PEFR and some anthropometric parameters between male and female students of 200 level MBBS/BDS students

\begin{tabular}{llll}
\hline Variables & Males (39) & Females (44) & P-value \\
\hline PEFR $(\mathrm{L} / \mathrm{min})$ & $491.79 \pm 67.19$ & $366.82 \pm 43.28$ & 0.00 \\
Trunk length $(\mathrm{cm})$ & $17.10 \pm 1.759$ & $16.77 \pm 1.461$ & 0.35 \\
Leg length $(\mathrm{cm})$ & $40.79 \pm 2.166$ & $38.36 \pm 2.48$ & 0.00 \\
Trunk-leg ratio & $0.42 \pm 0.06$ & $0.43 \pm 0.50$ & 0.09 \\
\hline \multicolumn{4}{r}{}
\end{tabular}

Table 3. Correlation between PEFR and some anthropometric parameters

\begin{tabular}{llll}
\hline Variables & No & R-value & P-value \\
\hline PEFR/Trunk-leg ratio & 83 & -0.107 & 0.33 \\
PEFR/Trunk length & 83 & 0.197 & 0.74 \\
PEFR/Leg length & 83 & $0.457^{* *}$ & 0.00 \\
\hline \multicolumn{4}{l}{ ( Correlation is significant at the 0.01 level (2-tailed) }
\end{tabular}

Table 4. Correlation of PEFR and some anthropometric parameters between gender

\begin{tabular}{lccc}
\hline Variables & No & R-value & P-value \\
\hline PEFR/Trunk-leg ratio & & & \\
Male & 39 & 0.198 & 0.227 \\
Female & 44 & 0.209 & 0.173 \\
PEFR/Trunk length & & & \\
Male & 39 & 0.348 & $0.030^{*}$ \\
Female & 44 & 0.330 & $0.029^{*}$ \\
PEFR/Leg length & & & \\
Male & 39 & 0.220 & 0.178 \\
Female & 44 & 0.154 & 0.317 \\
& *Correlation is significant at the 0.05 level (2-tailed)
\end{tabular}

Table 3 shows a significant positive correlation between PEFR and the leg length of the participants, no significant correlation between PEFR and trunk length but also shows a negative correlation between PEFR and the trunk-leg ratio although the relation is not statistically significant. while there is a significant (0.030) positive correlation between PEFR and Trunk length in both males and females which signified that the longer the trunk length the higher the PEFR (Table 4).

\section{CONCLUSION}

The results of this study revealed significant correlation between leg length and PEFR among the males and females, also revealed significant difference between the leg length and PEFR 
between the males and females. However, no significant relationship was established between the Trunk-leg ratio and PEFR both in the males and females.

\section{RECOMMENDATION}

It is recommended that further studies should look into relationship between PEFR and more anthropometric parameters and also consider children. A reference range for the Trunk-leg ratio in Nigeria should also be a future study.

\section{CONSENT}

As per international standard or university standard written participant consent has been collected and preserved by the authors.

\section{ETHICAL APPROVAL}

As per international standard written ethical permission has been collected and preserved by the authors.

\section{COMPETING INTERESTS}

Authors have declared that no competing interests exist.

\section{REFERENCES}

1. Al-Riyami BM, Al-Rawas OA, Hassan MO. Normal spirometric reference values for Omani children and adolescents, Respiratory. 2004;9(3):387-91.

2. Kim KS, Kwon OY, Yi CH. Effect of abdominal drawing-in maneuver on peak expiratory flow, forced expiratory volume in 1 second and pain during forced expiratory pulmonary function test in patients with chronic low back pain. Phys Ther Korea. 2009;16:10-17.

3. Gibson PG. Monitoring the patient with asthma: An evidence-based approach. J Allergy Clin Immunol. Medline. 2000;1:1726.

4. Eid N, Yandell B, Howell L, Eddy M, Sheikh S. Can peak expiratory flow predict airflow obstruction in children with asthma? Pediatrics. Medline. 2000;105(2): 354-8.

5. Minghelli B, Nunes C, Oliveira R. Prevalence of overweight and obesity in portuguese adolescents: Comparison of different anthropometric methods. North Am J Med Sci. 2013;5:653-9.

6. Bhogal S, Zemek R, Ducharme FM. Written action plans for asthma in children. Cochrane Database Syst Rev. 2006;21(3): 23-25.

7. Joseph P. Wilson, Alka M. Kanaya, Bo Fan, John A. Shephard. Ratio of Trunk to leg volume as a new body shape metric for diabetes and mortality. Plos One. 2013;8: $1-11$.

8. Ducher G, Jaffré C, Arlettaz A, Benhamou CL, Courteix D. Effects of longterm tennis playing on the muscle-bone relationship in the dominant and nondominant forearms. Can J Appl Physiol. 2005;30(1):3-17.

9. Ischander $M$, Zaldivar $F$ Jr, Eliakim $A$, Nussbaum E, Dunton G, Leu SY, et al. Physical activity, growth, and inflammatory mediators in BMI-matched female adolescents. Med Sci Sports Exerc. 2007; 39:1131-8.

10. Gracia-Marco L, Vicente-Rodriguez G, Casajus JA, Molnar D, Castillo MJ, Moreno LA. Effect of fitness and physical activity on bone mass in adolescents: The HELENA study. Eur J Appl Physiol. 2011; 111:2671-80

11. Fragoso CA, Gahbauer EA, Van Ness PH, Concato J, Gill TM. Peak expiratory flow as a predictor of subsequent disability and death in community-living older persons. J Am Geriatr Soc. 2008;56:1014-20.

12. Baptista F, Barrigas C, Vieira F, SantaClara H, Homens PM, Fragoso I, Sardinha LB. The role of lean body mass and physical activity in bone health in children. J Bone Miner Metab. 2012; 30(1):100-8.

13. Douma WR, van der Mark TW, Folgering HT. Mini-Wright peak flow meters are reliable after 5 years use. Eur Respir J. 1997;10:457-9.

14. Quanjer PH, Tammeling GJ, Cotes JE, Pedersen OF, Peslin R, Yernault JC. Lung volumes and forced ventilator flows; 1993 Update. Report working party "standardization of lung function tests", European coal and steel community, and European Respiratory Society. Eur Respir J. 1993. 6(Suppl. 16):5-40.

15. Bin Dong, Zhiqiang Wang, Jun Ma. Leg-totrunk ratio and the risk of hypertension in children and adolescents: A population- 
based study. Journal of Public Health. 2016;38:688-695.

16. Van de Wal BW, Erasnus LD, Hodler R. Sitting and standing heights in Blacks and white South Africans- The significans in relation to pulmonary function values. South African Medical Journal. 1971; 45(Suppl.):568-70.

(c) 2018 Adama et al.; This is an Open Access article distributed under the terms of the Creative Commons Attribution License (http://creativecommons.org/licenses/by/4.0), which permits unrestricted use, distribution, and reproduction in any medium, provided the original work is properly cited.

Peer-review history:

The peer review history for this paper can be accessed here: http://www.sdiarticle3.com/review-history/47410 